\title{
XML2NN: A Unified Modeling Method Accelerated by Distributed Training with an XML File
}

\author{
Mingxue Liao \\ Institute of Automation, Chinese Academy of Sciences \\ Haidian, Beijing, China \\ mingxue.liao@ia.ac.cn \\ Jiaxin Gao \\ Institute of Automation, Chinese Academy of Sciences \\ Haidian, Beijing, China \\ jiaxin.gao@ia.ac.cn
}

\begin{abstract}
Deep learning community in the computer vision field shows a trend of significantly expanding the scale of models and data. To ensure training and deployment efficiency, fast and effective modeling and distributed parallel training have become essential needs. However, there are various existing deep learning frameworks such as TensorFlow, Pytorch, Caffe, etc., which have different characteristics and programming syntax. This status quo hinders the progress of efficient modeling and model sharing. Meanwhile, these frameworks do not provide easy-to-use distributed training functions. To solve the above problems, in this paper, we introduce XML2NN, a unified modeling method for CV field with distributed training functions. With just an XML file, complex neural network construction, hyper-parameters setting and distributed parameters setting can be completed by users. XML2NN is based on multiple deep learning frameworks, which enables easy model construction and quick cross-framework model sharing. Extensive experiments on two CV tasks object detection and image classification have verified the modeling effectiveness and parallel performance of XML2NN.
\end{abstract}

\section{CCS CONCEPTS}

- Computing methodologies $\rightarrow$ Artificial and Intelligence.

\section{KEYWORDS}

Deep learning, Computer vision, Distributed training

\section{ACM Reference Format:}

Mingxue Liao, Haoyu Liang, Jiaxin Gao, and Pin Lv. 2021. XML2NN: A Unified Modeling Method Accelerated by Distributed Training with an XML File. In ACAI '2021: 4th International Conference on Algorithms, Computing and Artificial Intelligence, December 22-24, 2021, Sanya, Chian. ACM, New York, NY, USA, 7 pages. https://doi.org/10.1145/3508546.3508626

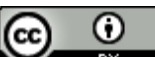

This work is licensed under a Creative Commons Attribution International 4.0 License.

ACAI '2021, December 22-24, 2021, Sanya, China

(C) 2021 Copyright held by the owner/author(s). Publication rights licensed to ACM. ACM ISBN 978-1-4503-8505-3/21/12.

https://doi.org/10.1145/3508546.3508626
}

\author{
Haoyu Liang \\ Institute of Automation, Chinese Academy of Sciences \\ Haidian, Beijing, China \\ haoyu.liang@ia.ac.cn \\ Pin Lv \\ Institute of Automation, Chinese Academy of Sciences \\ Haidian, Beijing, China \\ pin.lv@ia.ac.cn
}

\section{INTRODUCTION}

The development of deep learning has greatly promoted the progress of computer vision in recent years[ $[4,8-10,14,16,18,20]$. Various deep learning frameworks such as TensorFlow[1], Pytorch[13], etc. have been developed and widely used in academia and industry. The semantic expression scopes of these frameworks are almost the same. However, they have diverse design patterns, which leads to differences in programming syntax and usage methods. Users usually need to choose to use different frameworks in different projects according to their characteristics and advantages. According to the survey, Pytorch is more favored by academia, while TensorFlow is more common in industrial deployment[7]. This status quo traps users in the quagmire of learning usage and distinguishing characteristics of different frameworks. As a foreseeable result, a barrier that hinders the interconnection ecology has been built. There have been no effective approach to unify different frameworks.

On the other hand, distributed parallel training of deep models is an important mode for deep learning to become practical. Unfortunately, today's popular deep learning frameworks are not yet friendly enough to support distributed training. Sufficient domain knowledge in distributed computing and complex configurations are required for the native distributed training of TensorFlow and Pytorch. Furthermore, their parallel performance cannot be guaranteed.

To address aforementioned pain points, in this paper, we propose XML2NN, which provides a unified modeling method with distributed training ability for $\mathrm{CV}$ (Computer vision) field. This technology allows users to break away from a specific deep learning framework, so as to decouple the users and the frameworks. So XML2NN enables users to focus on model architecture and parameter adjustments. XML2NN builds on two optional backend engines, TensorFlow and Pytorch, which means cross-frame model sharing can be achieved with one-time model construction. Moreover, XML2NN is cluster-aware and provide pretty simple distributed training interface for users.

Due to the structured, platform-independent and checkability characteristics of XML (Extensible Markup Language), we use XML files for neural network model construction, training parameter setting and distributed setting. A schematic diagram of an example of building a neural network through an XML file is shown in Figure 1. To emphasize, our technical contributions are highlighted as follows: 


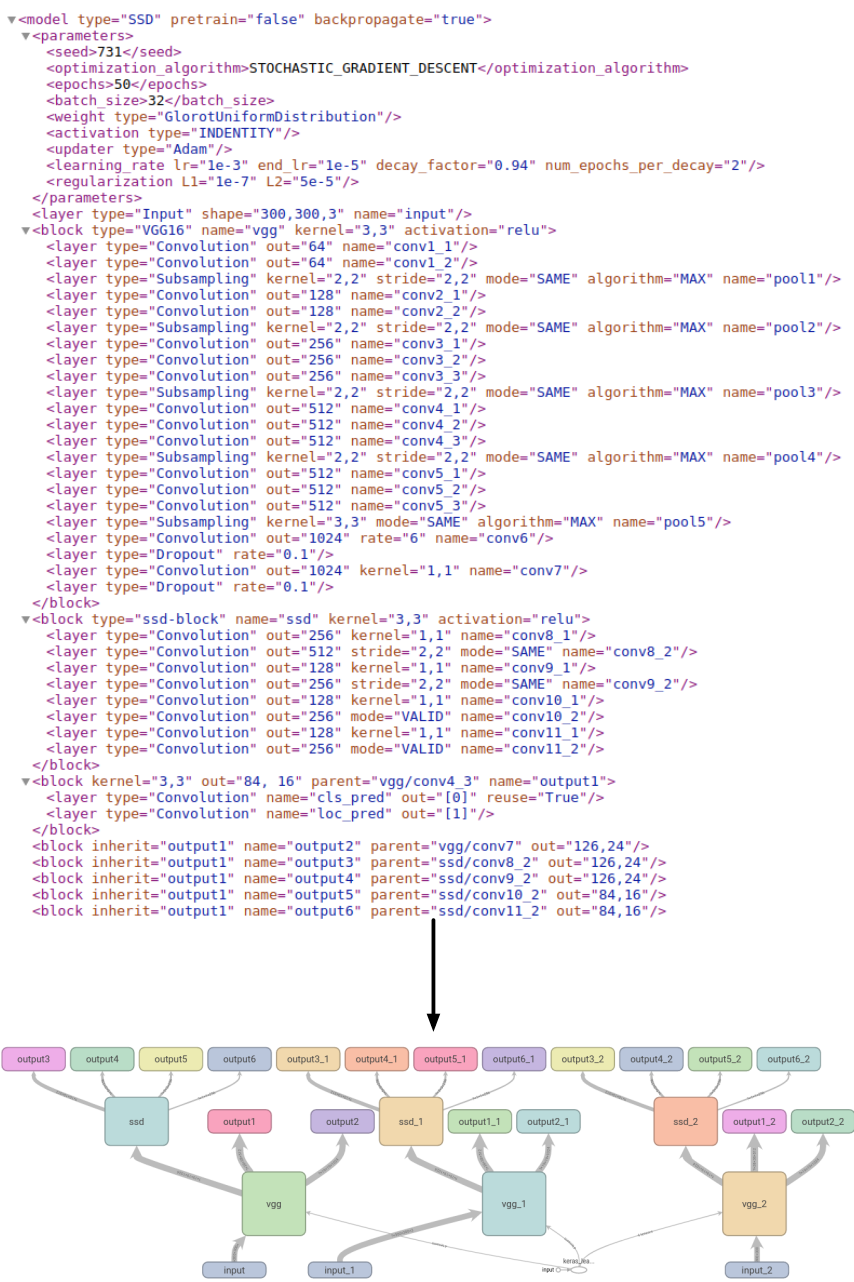

Figure 1: A schematic diagram of constructing an $\operatorname{SSD}$ [11] neural network through an XML file in XML2NN.

Easy-to-use unified modeling method. We provide pretty simple yet comprehensive model construction syntax . Considering that there are some differences in the usage between frameworks, we have done as much compatibility as possible and unify the interfaces of TensorFlow and Pytorch. Since module stacking has become a common paradigm in deep learning, we design block inherit mechanism to support stacking and free combination of network modules.

Cross-frame model sharing. TensorFlow and Pytorch serve as backend engines in XML2NN. Also benefit from its highly unified interfaces, XML2NN achieve the target of "One-time Construction, Everywhere Available", which will promote cross-framework model sharing among AI community. Once users complete the construction of a model in the XML file, the file can be embedded in different frameworks.

Distributed model training. XML2NN is cluster-aware, which means users only need to set the IP addresses of machines and the number of GPUs. In terms of parallel strategy, the synchronous update strategy based on ring all-reduce[2] is adopted. Under this strategy, the communication cost of synchronization is irrelevant to the number of machines, so this method has strong scalability.

\section{RELATED WORK}

Various frameworks continue to emerge with the rise of deep learning in the last decade. The design patterns and usage of these frameworks are diverse. For instance, TensorFlow1 and Pytorch define the calculation graph in different ways. TensorFlow1 builds neural networks through a static graph, which means users first define a calculation graph and then continuously feed data to the fixed graph to run the model. In Pytorch, the definition and the running of the calculation graph happen at the same time. As a result, TensorFlow1 has a faster running speed but is more troublesome to debug for users, while Pytorch is more user-friendly in programming and debugging. The existence of differences allows users to use the most suitable framework according to their needs, but also requires users to be familiar with the usage of multiple frameworks. Moreover, it resulted in lots of time wasted in migrating one model from one framework to another.

Based on above problems, there have been some attempts to provide a cross-platform intermediate representation method. ONNX is released by Microsoft and Facebook, which aims to enable neural network models to be shared among different frameworks. However, ONNX can only converted a constructed model based on a certain framework into an intermediate representation defined by ONNX. Users can not directly construct a neural network model from scratch using ONNX. In addition, ONNX also faces the problems of poor readability and low transfer success rate. In NLP(Natural Language Processing) field, NeuronBlocks[6] is an open source toolkit that allows users to build NLP models quickly and flexibly using JSON files. But NeuronBlocks only builds on the Pytorch backend engine and does not support other frameworks, so it can hardly realize cross-framework model sharing. Besides, distributed parallel training function is not supported in NeuronBlocks.

\section{DESIGN}

\subsection{Overview}

To train a neural network, the following three steps are essential. The first is basic construction of neural network structure, and the second is the preprocessing of the dataset, and the third is the setting of training parameters. XML2NN provides convenient interfaces for completing all these three steps. For model construction, XML2NN supports complete and easy-to-use interfaces for various operators, networks, and other modules. These functions are described in 3.2 and 3.3. For dataset preprocessing, some general preprocessing method templates are provided. In addition, users can customize the preprocessing method and reference it in the XML file. This module is introduced in 3.5. For training parameter settings, as XML2NN is cluster-aware, in addition to basic model training parameters, distributed training parameter settings are also provided. This module is introduced in 3.4. Based on these functions, we provides bottom-up support from operators, networks, general CV models, until general CV tasks in XML2NN.

XML2NN parses the user input file and starts the training of the neural networks through the following steps. First, the model 


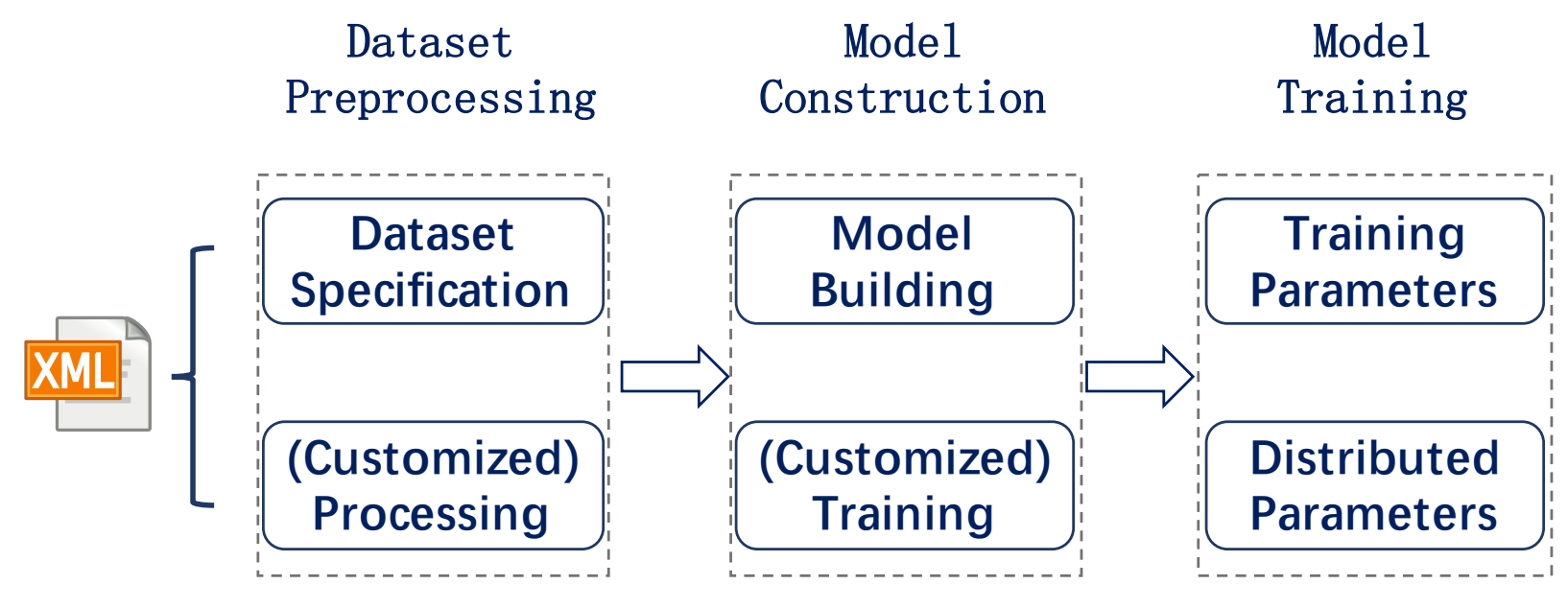

Figure 2: The processing flow of XML2NN to start a model training. It is comprised of three main steps: dataset preprocessing, model construction and model training.

training parameters are parsed and stored for subsequent use. Then the dataset specified by the user is loaded, and preprocessing is performed using the built-in or customized data preprocessing method. Next, the network structure is parsed and a data flow diagram is generated. According to the backend engine specified by the user, the data flow diagram is adapted to the Pytorch dynamic graph or the TensorFlow graph. Finally, start the model training combining with the training parameters. In addition, if distributed training parameters are specified, the distributed training engine will be started before starting training. The overview of XML2NN system is depicted in Figure 2.

\subsection{Supported Functions}

In XML2NN, most basic operators and network layers used in CV field are supported: Convolutional layer(2D, 3D), pooling layer, padding layer, batch normalization layer, dropout layer, up-sampling layer, residual connection, various element-wise operations, various tensor operations(such as flatten, squeeze, reshape), various mathematical calculation operations. In addition, the following modules are also provided:

Attention module. Three attention mechanisms[12] are provided: additive attention mechanism, dot multiplication attention mechanism and dense attention mechanism.

Activation function. All built-in activation function layers in TensorFlow/Pytorch are supported, including ReLU, Sigmoid, Softmax and etc.

Loss function. Log-likelihood loss, cross-entropy loss, square loss, hinge loss, triplet loss and etc. are supported. For the reason that the final loss in many tasks are composed of different types of loss functions, we also support the assembly of different loss functions and assign a weighting factor to each one.

Parameter initialization. All built-in parameter initialization methods in TensorFlow/Pytorch are supported, including random uniform initialization, random normal initialization, orthogonal initialization, Xavier initialization[5] and etc. We provide fine-grained initialization control. For instance, users can specify the mean and variance when using random normal initializati on.

Optimizer. All optimizers supported by TensorFlow/Pytorch are provided in XML2NN. At the same time, we support fine grained control of learning rate. Users can specify parameters such as the initial learning rate, the minimum learning rate, and the learning rate decay rate.

Evaluation metric. For classification task, accuracy, precision rate, recall rate and F1 value are supported. For object detection task, mAP is supported. For image segmentation task, pixel accuracy, IoU and etc. are supported.

\subsection{Model Construction}

XML2NN uses the syntax of XML to cover the semantic range required to build the model, while ensuring that there is no ambiguity. Next, we briefly introduce the construction grammar of the model.

Model structure, parameters and dataset are defined in XML file. $<$ model $>$ is the root element of the file, whose attribute "platform" specifies which deep learning framework is used as backend engine. The specification of the dataset and the related dataset parameters setting are completed in the first sub-element $<$ dataset $>$. The setting of hyper-parameters is completed in the second sub-element $<$ parameters $>$. Subsequently, the sub-elements $<$ layer $>$, $<$ vertex $>$ and $<$ block $>$ that can be reused complete the model construction.

$<$ layer > represents two types of components. One is the neural network layers including convolutional layer, fully-connected layer, pooling layer, dropout layer, etc. The other is the operators that operate on one input vector, including reshape operation, squeeze operation, etc. <layer $>$ is the main component of the network.

$<$ vertex $>$ represents the operators that operate on two or more input vectors, including concatenation operation, element-wise operators, etc. 
$<$ block $>$ represents a network block that contains several subelements of $<$ layer $>$ and $<$ vertex $>$. $<$ block $>$ can be inherited to realize flexible reuse of modules. For the reason that many modules are often stacked in CV field, block inheritance mechanism allows users to flexibly stack and reuse their custom blocks.

Data flow control. In the process of model construction, how to control the flow of data on the network must be considered. It is planned to specify the input data of each layer through the "parent" attribute, allowing users to flexibly control the data flow. This method is more intuitive and has better legibility. Figure 1 shows a sample XML file for constructing an SSD model in the object detection task, and the visualization of the model's calculation graph.

General model template(model zoo). We provide templates of some general model in the $\mathrm{CV}$ field to facilitate users to train these models directly in XML2NN without the need for model construction. At the same time, the data preprocessing templates corresponding to these models will also be provided. This means that users only need to provide their own training setting and specify the parameters to directly start the training of the model.

\subsection{Parallel Ability}

Based on data parallel of Horovod distributed framework, XML2NN provides users with highly encapsulated distributed training services. The Horovod uses a ring based communication architecture, whose communication cost is unrelated with the number of GPUs. Therefore, although a strict synchronization update strategy is used, the scalability will not be affected.

XML2NN is cluster-aware. If users have established their cluster environment, then they do not need to perform tedious resource configuration and parallel management, and only need to specify the IP of machines and number of GPUs used in the XML file to start one-click distributed training. The schematic diagram is shown in Figure 3.

\subsection{Task Customization}

The complete operation process of training a deep learning model includes dataset preprocessing, model construction and model training. For some tasks that require complex dataset preprocessing and other training steps, fine-grained operations are needed. If users create these operations from scratch in an XML file, it will consume a lot of time and effort on code details. At the same time, these operations are usually reusable. Based on this, we provide standardized dataset preprocessing method. Meanwhile, users can also custom their own methods and then refer them in XML files.

To be specific, taking the Yolov3 model in the object detection task as an example, this task requires anchor generation, boxes filtering, and more complex data preprocessing during training. We provide the standardized interface of these operations, and users can specify the operations in XML files. Combined with Model zoo, XML2NN realizes comprehensive support for many common tasks and models.

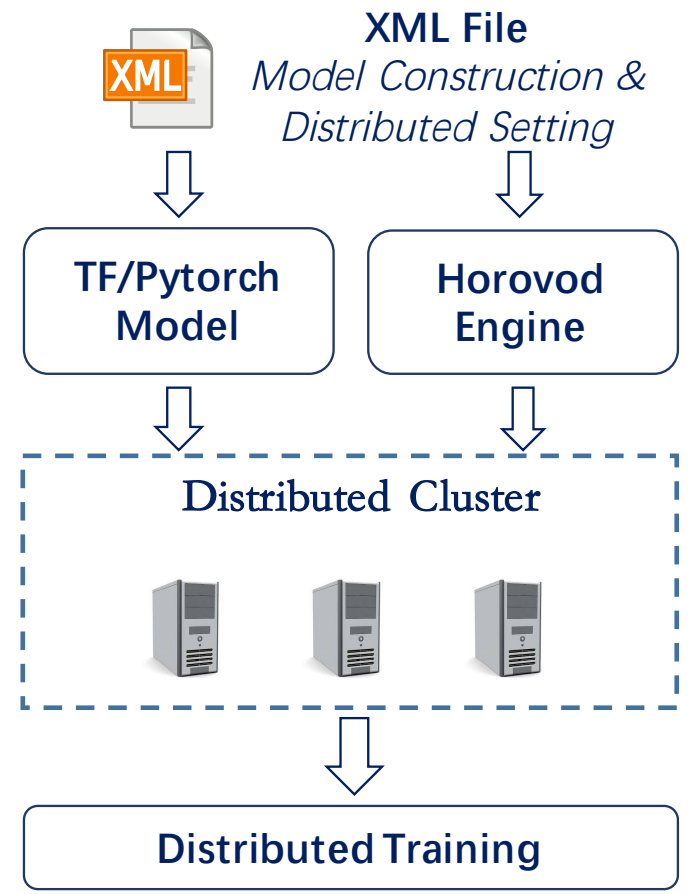

Figure 3: A schematic diagram of starting a distributed parallel training in XML2NN.

\section{EXPERIMENTS}

To verify the effectiveness and performance of XML2NN, we conduct experiment reports on two aspects of results: modeling effectiveness and parallel performance. For model effectiveness, multiple popular models in object detection task are evaluated. For parallel performance, several classical $\mathrm{CNN}$-based models for image classification are evaluated. Our experiment platform is based on a machine cluster, which includes five servers. Among them, there are four servers each equipped with one GPU, and one server equipped with four GPUs. All eight GPUs are NVIDIA GeForce 2080 Ti with $11 \mathrm{~GB}$ memory.

\subsection{Modeling Effectiveness}

We verify the effectiveness of XML2NN modeling through the construction of several object detection models. The reason for choosing the object detection models is that the object detection task is one of the core tasks in the CV field. Deep learning methods have made eye-catching progress in stages in this task, and have been widely deployed in the industry. Moreover, the models usually have complex structures, which can test and reflect the effectiveness of modeling with XML2NN.

Baseline Methods. Three types of models: Yolo-v3[15], Yolo-v4[3] and SSD300[11] are chosen, which are at the top of the current object detection community. They are modeled in two ways respectively: our XML2NN method and ordinary method with Pytorch. 


\begin{tabular}{lrr}
\hline Model & $\mathrm{mAP}(\%)$ & Training Time per Epoch(s) \\
\hline SSD300(TensorFlow) & 75.6 & 927.8 \\
SSD300(XML2NN) & 75.7 & 927.0 \\
\hline Yolo-v3-320(TensorFlow) & 79.9 & 490.8 \\
Yolo-v3-320(XML2NN) & 80.3 & 492.1 \\
\hline Yolo-v4(Pytorch) & 84.7 & 341.4 \\
Yolo-v4(XML2NN) & 84.5 & 338.9
\end{tabular}

Table 1: Performance comparison of XML2NN models and Pytorch models on three methods: Yolo-v3, Yolo-v4 and SSD. Models constructed by XML2NN have same performance with ordinary modeling models.

\begin{tabular}{lrrr}
\hline GPU numbers & mAP & Total Training Time(h) & Speedup Ratio(\%) \\
\hline 1 & 75.7 & 927.0 & 100.0 \\
2 & 75.3 & 536.7 & 86.5 \\
4 & 74.7 & 312.8 & 74.1 \\
\hline
\end{tabular}

Table 2: Performance comparison in parallel training mode on three models: Yolo-v3, Yolo-v4 and SSD. SSD has best acceleration performance.

\begin{tabular}{cccccc}
\hline Model & Input Size & Params(MB) & Feat(MB) & GPU numbers & Throughput (images/s) \\
\hline \multirow{5}{*}{ AlexNet } & $227 \times 227$ & 233 & 3 & 1 & 3695.84 \\
& $227 \times 227$ & 233 & 3 & 2 & 6312.63 \\
& $227 \times 227$ & 233 & 3 & 3 & 7857.38 \\
& $227 \times 227$ & 233 & 3 & 4 & 8153.95 \\
\hline \multirow{5}{*}{ VGG16 } & $224 \times 224$ & 548 & 63 & 1 & 177.60 \\
& $224 \times 224$ & 548 & 63 & 2 & 294.05 \\
& $224 \times 224$ & 548 & 63 & 3 & 344.96 \\
& $224 \times 224$ & 548 & 63 & 4 & 385.41 \\
\hline \multirow{5}{*}{ ResNeption3 } & $299 \times 299$ & 91 & 89 & 1 & 199.52 \\
& $299 \times 299$ & 91 & 89 & 2 & 377.21 \\
& $299 \times 299$ & 91 & 89 & 3 & 526.12 \\
& $299 \times 299$ & 91 & 89 & 4 & 695.96 \\
\hline \multirow{5}{*}{ ResNet152 } & $224 \times 224$ & 98 & 103 & 1 & 301.60 \\
& $224 \times 224$ & 98 & 103 & 2 & 572.58 \\
& $224 \times 224$ & 98 & 103 & 3 & 777.44 \\
& $224 \times 224$ & 98 & 103 & 4 & 951.21 \\
\hline & $224 \times 224$ & 230 & 219 & 2 & 113.89 \\
& $224 \times 224$ & 230 & 219 & 4 & 213.60 \\
\hline
\end{tabular}

Table 3: Throughout comparison on several CNN models: AlexNet, VGG16, Inception4, ResNet50 and ResNet152.

The result we expect is that the models by XML2NN achieve indistinguishable results compared with the ordinary modeling models.

- Yolo-v3. Yolo family is the pioneer of the one-stage method. Yolo-v3 and Yolo-v4 are the latest and mature versions in the family. Yolo-v3 uses Darknet53 as the backbone network. Darknet53 has similar accuracy to ResNet101 and has faster speed. Yolo-v3 adopts multi-scale detection, which effectively improves the detection accuracy of small targets.

- Yolo-v4. Yolo-v4 uses CSPDarknet53 with more parameters as backbone network. SPP module is added to the network to increase the receptive field. Yolo-v4 integrates a series of training tricks, and has the highest accuracy among all 
current real-time object detection methods. It also achieves the best trade-off in model accuracy and detection speed.

- SSD. SSD first combines the regression method in Yolo family models and the anchor mechanism in Faster-RCNN[16], which not only keeps the accuracy of Faster-RCNN in boxes predictions, but also has the characteristics of fast calculation speed. SSD adopts the idea of multi-scale detection, extracting multiple feature maps of different scales for detection.

Training Settings. The experiments are conducted on PASCAL VOC dataset, which have twenty classes. We train our models on VOC2007 +2012 (train and val), which contains 16551 samples. Then we test our models, and report the $\mathrm{mAP}$ (mean Average Precision) on VOC2007 test, which contains 4952 images. For SSD300, we use Momentum SGD optimizer with 0.9 momentum and $5 \mathrm{e}-4$ weight decay. The batch size is set to 48 . The model is first trained for $25 \mathrm{k}$ steps with learning rate $1 \mathrm{e}-3$, and then trained for $10 \mathrm{k}$ steps with learning rate $1 \mathrm{e}-4$, and finally trained for $15 \mathrm{k}$ steps with learning rate $1 \mathrm{e}-5$ For Yolo-v3, we use Adam optimizer with 5e-4 weight decay. The batch size is set to 64 . The model is trained for 100 epochs. The learning rate decays from $1 \mathrm{e}-3$ to $1 \mathrm{e}-5$ with the training process. For Yolo-v4, we use Adam optimizer with 5e-4 weight decay. The batch size is set to 64 . The model is trained for 300 epochs with learning rate $2.61 \mathrm{e}-3$.

Result Analysis. mAP(mean Average Precision) results on test set are reported in Table 1. Compared with models built with ordinary method, the models built using XML2NN achieved competitive performance.

In addition, to further study modeling effectiveness under distributed training mode, we also provide distributed training performance of above models. We conduct experiments using 1, 2 and 4 GPUs. As for the parallel strategy, synchronous data parallel method based on ring all-reduce is chosen. The results are shown in Table 2. When using multiple GPUs, the mAP results of three models are lossless compared with one-gpu training models. In terms of parallel acceleration, the three models achieve good acceleration effect. SSD achieves the highest speedup, and the results of two Yolo models are similar. Since Yolo-v3 and Yolo-v4 have more parameters than SSD, they need more communication time to exchange parameters. The speedup ratios on these two models are a little lower than SSD.

\subsection{Parallel Performance}

To verify the parallel performance of the XML2NN model, we test the throughput of several general CNN models for processing images. We choose AlexNet[10], VGG16[17], Inception3[19], Inception4, ResNet50[9] and ResNet152[9] that have different model structures and different scale parameters. These models are common backbone networks in CV field, and are adopted as upstream support for various CV tasks. So it can be said that the training efficiency on these networks is crucial.

Training Settings. All models are trained on ImageNet dataset. All models are optimized using Adam optimizer with 1e-3 learning rate. The batch sizes of AlexNet, VGG16, Inception3, Inception4, ResNet50, ResNet152 are set to 512, 64, 64, 16, 64, 32. For each model, we use 1, 2, and 4 GPUs on one machine for experiments. It is worth mentioning that since we focus on model throughput in this subsection and the training time of ImageNet is too long, we have not trained all models to convergence.

Result Analysis. The results are shown in Table 3.First of all, it can be seen that because these models have obvious differences in model structure, width, and depth, their throughput also shows very obvious differences. Among them, the AlexNet model has the simplest structure and the smallest depth, so the throughput is an order of magnitude larger than other models. The ResNet-152 model has the largest depth, so the memory occupied by the model features is the largest, and the throughput is the smallest among all models.

Next, we focus on the throughput performance of each model on multiple GPUs. In summary, several models have achieved good acceleration results. Among them, ResNet-152 has the best acceleration effect. Its acceleration ratio reached $93.7 \%$ in two GPUs and $78.7 \%$ in four GPUs. The lowest AlexNet also has $85.4 \%$ speedup in two GPUs, and $55.2 \%$ speedup in four GPUs. This verifies the effectiveness of the parallel strategy in XML2NN. Theoretically, if the parameters of the two models are equal, when a model requires the longer calculation time to process a single image under nonparallel conditions, its acceleration effect under data parallelism is better. Because under data parallelism, because the parameters of the two models are the same, they need the same model parameter communication time, so the ratio of the calculation time to the communication time of the model with a longer calculation time is greater than that of the other model, and its speedup ratio is higher. Among the several models used in the experiment, ResNet-152 has the longest single image calculation time, and its speedup is the highest among all models, and its throughput increases almost linearly with the increase of the number of GPUs. In contrast, AlexNet and ResNet-152 have basically the same parameter amount, and the calculation time of a single image is only one-32 times that of ResNet-152, and its speedup is much lower than ResNet-152, only $74 \%$.

\section{CONCLUSION AND FUTURE WORK}

In this paper, we propose XML2NN, which provide a unified modeling method with distributed training ability for $\mathrm{CV}$ (Computer vision) field. This technology allows users to break away from a specific deep learning framework, so as to decouple the users and the framework. We use XML format files for neural network model building, parameter setting and dataset designation. In XML file, model construction, model training and cross-frame model sharing. To emphasize, we provide Easy-to-use model building method, cross-frame model sharing ability and Distributed model training function. Extensive experiments on two CV tasks object detection and image classification have verified the modeling effectiveness and parallel performance of XML2NN.

In future work, we will apply our XML2NN method to more deep learning fields, e.g. NLP(Natural Language Processing). In addition, We will also try to support richer parallel strategies, such as model parallelism. Because the scale of today's deep learning models is showing an increasing trend, a single GPU cannot install a large model in many cases. At this time, a model parallel strategy is needed. 


\section{REFERENCES}

[1] Martín Abadi, Paul Barham, Jianmin Chen, Zhifeng Chen, Andy Davis, Jeffrey Dean, Matthieu Devin, Sanjay Ghemawat, Geoffrey Irving, Michael Isard, Manjunath Kudlur, Josh Levenberg, Rajat Monga, Sherry Moore, Derek G. Murray, Benoit Steiner, Paul Tucker, Vijay Vasudevan, Pete Warden, Martin Wicke, Yuan $\mathrm{Yu}$, and Xiaoqiang Zheng. 2016. TensorFlow: a system for large-scale machine learning. In OSDI'16 Proceedings of the 12th USENIX conference on Operating Systems Design and Implementation. 265-283.

[2] Gibiansky Andrew. 2017. Bringing HPC Techniques to Deep Learning. https: //andrew.gibiansky.com/blog/machine-learning/baidu-allreduce/. (2017).

[3] Alexey Bochkovskiy, Chien-Yao Wang, and Hong-Yuan Mark Liao. 2020. YOLOv4: Optimal Speed and Accuracy of Object Detection. arXiv preprint arXiv:2004.10934 (2020).

[4] L. A. Gatys, A. S. Ecker, and M. Bethge. 2016. Image Style Transfer Using Convolutional Neural Networks. In 2016 IEEE Conference on Computer Vision and Pattern Recognition (CVPR).

[5] Xavier Glorot and Yoshua Bengio. 2010. Understanding the difficulty of training deep feedforward neural networks. In Proceedings of the Thirteenth International Conference on Artificial Intelligence and Statistics (Proceedings of Machine Learning Research, Vol. 9), Yee Whye Teh and Mike Titterington (Eds.). PMLR, Chia Laguna Resort, Sardinia, Italy, 249-256. http://proceedings.mlr.press/v9/glorot10a.html

[6] M. Gong, L. Shou, W. Lin, Z. Sang, Q. Yan, Z. Yang, F. Cheng, and D. Jiang. 2019. NeuronBlocks: Building Your NLP DNN Models Like Playing Lego.

[7] Horace He. 2019. The State of Machine Learning Frameworks in 2019. https://thegradient.pub/state-of-ml-frameworks-2019-pytorch-dominatesresearch-tensorflow-dominates-industry/. The Gradient (2019).

[8] K. He, G. Gkioxari, P Dollár, and R. Girshick. 2017. Mask R-CNN. In IEEE.

[9] Kaiming He, Xiangyu Zhang, Shaoqing Ren, and Jian Sun. 2016. Deep Residual Learning for Image Recognition. In 2016 IEEE Conference on Computer Vision and Pattern Recognition (CVPR).

[10] Krizhevsky, Alex, Sutskever, Ilya, Hinton, and E. Geoffrey. 2012. ImageNet Classification with Deep Convolutional Neural Networks. Advances in neural information processing systems (2012).
[11] Wei Liu, Dragomir Anguelov, Dumitru Erhan, Christian Szegedy, Scott E. Reed, Cheng-Yang Fu, and Alexander C. Berg. 2016. SSD: Single Shot MultiBox Detector. european conference on computer vision (2016), 21-37.

[12] Thang Luong, Hieu Pham, and Christopher D. Manning. 2015. Effective Approaches to Attention-based Neural Machine Translation. In Proceedings of the 2015 Conference on Empirical Methods in Natural Language Processing. Association for Computational Linguistics, Lisbon, Portugal, 1412-1421. https: //doi.org/10.18653/v1/D15-1166

[13] Adam Paszke, Sam Gross, Francisco Massa, Adam Lerer, James Bradbury, Gregory Chanan, Trevor Killeen, Zeming Lin, Natalia Gimelshein, Luca Antiga, Alban Desmaison, Andreas Köpf, Edward Yang, Zach DeVito, Martin Raison, Alykhan Tejani, Sasank Chilamkurthy, Benoit Steiner, Lu Fang, Junjie Bai, and Soumith Chintala. 2019. PyTorch: An Imperative Style, High-Performance Deep Learning Library. arXiv preprint arXiv:1912.01703 (2019).

[14] Joseph Redmon, Santosh Divvala, Ross Girshick, and Ali Farhadi. 2016. You Only Look Once: Unified, Real-Time Object Detection. In Computer Vision \& Pattern Recognition.

[15] Joseph Redmon and Ali Farhadi. 2018. YOLOv3: An Incremental Improvement. arXiv preprint arXiv:1804.02767 (2018).

[16] Shaoqing Ren, Kaiming He, Ross Girshick, and Jian Sun. 2015. Faster R-CNN: Towards Real-Time Object Detection with Region Proposal Networks. IEEE Transactions on Pattern Analysis and Machine Intelligence 39, 6 (2015).

[17] K. Simonyan and A. Zisserman. 2015. Very Deep Convolutional Networks for Large-Scale Image Recognition. In International Conference on Learning Representations.

[18] Christian Szegedy, Wei Liu, Yangqing Jia, Pierre Sermanet, Scott Reed, Dragomir Anguelov, Dumitru Erhan, Vincent Vanhoucke, and Andrew Rabinovich. 2014. Going Deeper with Convolutions. (2014).

[19] Christian Szegedy, Vincent Vanhoucke, Sergey Ioffe, Jonathon Shlens, and Zbigniew Wojna. 2015. Rethinking the Inception Architecture for Computer Vision. arXiv preprint arXiv:1512.00567 (2015).

[20] Oriol Vinyals, Alexander Toshev, Samy Bengio, and Dumitru Erhan. 2015. Show and tell: A neural image caption generator. In 2015 IEEE Conference on Computer Vision and Pattern Recognition (CVPR). 3156-3164. https://doi.org/10.1109/CVPR. 2015.7298935 